\title{
Response and Long-Term Control of Bone Metastases After Peptide Receptor Radionuclide Therapy with ${ }^{177}$ Lu-Octreotate
}

\author{
Samer Ezziddin ${ }^{1}$, Amir Sabet ${ }^{1}$, Florian Heinemann ${ }^{1}$, Charlotte J. Yong-Hing ${ }^{2}$, Hojjat Ahmadzadehfar ${ }^{1}$, Stefan Guhlke ${ }^{1}$, \\ Tobias Höller ${ }^{3}$, Winfried Willinek ${ }^{4}$, Christian Boy ${ }^{5}$, and Hans-Jürgen Biersack ${ }^{1}$ \\ ${ }^{1}$ Department of Nuclear Medicine, University Hospital, Bonn, Germany; ${ }^{2}$ Department of Radiology, University of British Columbia \\ Hospital, Vancouver, British Columbia, Canada; ${ }^{3}$ Institute of Medical Biometry, Informatics and Epidemiology, Bonn, Germany; \\ ${ }^{4}$ Department of Radiology, University Hospital, Bonn, Germany; and ${ }^{5}$ Department of Nuclear Medicine, University Hospital, \\ Essen, Germany
}

Peptide receptor radionuclide therapy (PRRT) is an efficient treatment for gastroenteropancreatic neuroendocrine tumors (GEP NETs), with outstanding overall response rates and survival. However, little is known about the particular efficacy regarding bone metastasis (BM). Methods: We retrospectively analyzed a consecutive subgroup of 42 patients with BM of GEP NETs treated with PRRT ( ${ }^{177} \mathrm{Lu}$-octreotate, 4 intended cycles at 3 monthly intervals [10-14 wk]; mean activity per cycle, $8.1 \mathrm{GBq}$ ). Availability of restaging and outcome data was required for patient inclusion. Baseline characteristics, including age, tumor origin, performance score, Ki-67 index, tumor load, tumor uptake, plasma chromogranin $A$, and neuron-specific enolase, were analyzed regarding impact on tumor regression (modified M.D. Anderson criteria) and time to progression. Survival analyses were performed using Kaplan-Meier curves, log-rank test at a significance level of $P$ less than 0.05, and Cox proportional hazards model for uni- and multivariate analyses. Results: Median follow-up was $32 \mathrm{mo}$. The observed response of BMs consisted of complete remission in $2(4.8 \%)$, partial remission in 14 (33.3\%), minor response in 5 (11.9\%), stable disease in 16 (38.1\%), and progressive disease in 5 (11.9\%) patients. Median progression-free survival and overall survival (OS) were $35 \mathrm{mo}$ (26-44, 95\% confidence interval) and $51 \mathrm{mo}$ (37-65, 95\% confidence interval), respectively. Patients with responding BMs (complete remission, partial remission, or minor response) exhibited a trend toward better OS (median OS not reached after $53 \mathrm{mo}$ ) when compared to nonresponding patients (39 mo, $P=0.076)$. Only Ki-67 index $(>10 \%)$ and chromogranin A level $(>600 \mathrm{ng} / \mathrm{mL})$ contributed to regression analysis. Conclusion: BM of GEP NETs is effectively controlled by PRRT, with long progression-free survival and OS. Poor patient condition and multifocality of BMs do not clearly affect treatment efficacy, possibly encouraging the use of PRRT in advanced bone metastatic disease. Larger studies are needed to assess predictors of treatment outcome in these patients.

Received Mar. 13, 2011; revision accepted May 16, 2011.

For correspondence or reprints contact: Samer Ezziddin, Department of Nuclear Medicine, University Hospital Bonn, Sigmund-Freud-Strasse 25, D-53105 Bonn, Germany.

E-mail: samer.ezziddin@ukb.uni-bonn.de

COPYRIGHT $\odot 2011$ by the Society of Nuclear Medicine, Inc.
Key Words: neuroendocrine tumors; bone metastases; peptide receptor radionuclide therapy; ${ }^{177} \mathrm{Lu}$-DOTA octreotate; oncology

J Nucl Med 2011; 52:1197-1203

DOI: 10.2967/jnumed.111.090373

$\mathbf{T}$ he therapeutic management of gastroenteropancreatic neuroendocrine tumors (GEP NETs) is challenging in the metastatic, unresectable stage. Bone metastases (BMs) are present in $8 \%-19 \%$ of metastatic GEP NETs (1-6). They predominantly occur in patients with liver metastases $(4,7,8)$, but bone-only disease has been observed. The clinical impact of BMs is significant; they cause pain and eventually decreased bone marrow function. However, apart from one study describing an impaired outcome in patients with nonhepatic distant metastases (2), there is little published data on the general prognostic impact of BM in NET $(1,9)$.

Few studies have examined the influence of BMs on outcome. In the context of peptide receptor radionuclide therapy (PRRT), one study reports a negative impact of the presence of BMs on time to progression after ${ }^{177} \mathrm{Lu}$-octreotate (10). PRRT is an efficient systemic treatment for metastatic GEP NETs, producing outstanding overall response and survival (11). It is safe even when readministered during relapse of progressive disease $(12,13)$. However, little is known about the particular efficacy of this treatment regarding BMs.

This retrospective study aims to assess the therapeutic effect of PRRT, namely with ${ }^{177} \mathrm{Lu}$-octreotate, on metastatic bone disease in a specific subgroup and identify potential risk factors for impaired outcome.

\section{MATERIALS AND METHODS}

\section{Patients}

Forty-two consecutive patients (mean age, $62 \mathrm{y}$; age range, 44-88 y; 26 men, 16 women) with BMs were retrospectively analyzed. All patients had well-differentiated GEP NETs, were treated with PRRT at our institution, and had their restaging completed. Twelve patients had pancreatic NET, and 30 patients had 
nonpancreatic GEP NETs, of which 4 had foregut, 9 midgut, 3 hindgut, and 12 other GEP NETs including an unknown primary. Eleven patients had metastatic bone pain. Apart from bone, metastatic sites included liver in 41 (97.6\%), lymph nodes in 25 $(59.5 \%)$, and other organs in $14(33.3 \%)$ patients. Previous treatments included surgery ( $n=22,52.4 \%)$, biotherapy $(n=17$, $40.5 \%)$, chemotherapy $(n=11,26.2 \%)$, and locoregional treatment $(n=2,4.8 \%)$.

\section{Histopathology}

Patients were classified according to the current TNM staging and grading system for NET $(14,15)$. All tumors were welldifferentiated endocrine carcinomas according to the histopathology with the presence of distant metastases (TNM stage IV). The histology and the Ki-67 proliferation index were determined out of resection specimens $(n=19 ; 45.2 \%)$ or biopsy material $(n=23 ; 54.8 \%)$ from liver metastases or the primary tumor. Immunohistochemical assessment of the Ki-67 index using the MIB1 antibody was expressed as percentage of stained tumor cells in 2,000 cells in areas in which the highest nuclear labeling was observed $(14,15)$.

\section{PRRT}

Inclusion criteria for treatment with PRRT were histologically confirmed, unresectable, metastatic GEP NETs; sufficient tumor uptake (i.e., $\geq$ liver uptake on baseline receptor imaging); a glomerular filtration rate of more than $30 \mathrm{~mL} / \mathrm{min} / 1.73 \mathrm{~m}^{2}$; a white blood cell count of $2 \times 10^{9} / \mathrm{L}$ or more; and platelets more than $70 \times$ $10^{9} / \mathrm{L}$. PRRT was performed by administration of $8.1 \pm 0.98 \mathrm{GBq}$ of ${ }^{177} \mathrm{Lu}$-DOTA octreotate per treatment cycle, aimed at 4 courses at standard intervals of $3 \mathrm{mo}(10-14 \mathrm{wk})$. The ${ }^{177} \mathrm{Lu}$ (IDB Holland) had a specific activity in the range of $100-160 \mathrm{GBq} / \mu \mathrm{mol}$ at the time of administration. Peptide labeling $(16,17)$ was performed to obtain apparent specific activity of about $54 \mathrm{GBq} / \mu \mathrm{mol}$ (ratio of activity to the total amount of peptide).

\section{Response Assessment}

Patients were restaged 3 mo after termination of PRRT. CT or MRI was performed according to the baseline imaging modality. Functional imaging was also performed consisting of somatostatin receptor scintigraphy $\left({ }^{111}\right.$ In DTPA-octreotide [OctreoScan]; Covidien) or somatostatin receptor PET/CT ( ${ }^{68} \mathrm{Ga}$-DOTATOC) and bone scintigraphy. Follow-up imaging was usually performed at 6-mo intervals after the first restaging.

Overall tumor response in our center was reported according to the modified Southwest Oncology Group (SWOG) solid tumor response criteria $(18,19)$, with minor response (MR) being defined as $25 \%-49 \%$ remission of the sum of products of perpendicular diameters of all measurable tumor lesions. Response of BMs, which classically reflect nonmeasurable disease in conventional imaging response criteria (response evaluation criteria in solid tumors [RECIST], SWOG), was determined according to functional M.D. Anderson criteria (20), modified for the purpose of assessment in NET. Complete remission (CR) was defined as complete resolution of all bone lesions in functional imaging; partial remission (PR) as complete disappearance of one or more bone lesions, together with substantial decrease in tracer uptake in the remaining lesions; MR as substantial decrease in tracer uptake in the bone lesions, without complete resolution of any lesion; stable disease as no significant change in functional imaging; and progressive disease (PD) as any new bone lesion.

\section{Outcome and Statistical Analysis}

The baseline characteristics of the study population were analyzed regarding the associated tumor response. For this purpose, the Fisher exact test was applied after dichotomizing for each factor and the resulting response: regression (CR, PR, or $\mathrm{MR}$ ) versus nonregression (stable disease or $\mathrm{PD}$ ) and progression (PD) versus nonprogression (CR, PR, MR, or stable disease).

Overall survival (OS) and progression-free survival were censored at the time of commencement of another significant treatment, such as chemotherapy or salvage PRRT, but not somatostatin medication. The underlying event for calculation of progression-free survival was documentation of progression of BMs. Survival analysis was performed using the Kaplan-Meier curve method. The log-rank test was carried out with a significance level of $P$ less than 0.05 . Univariate regression analysis by Cox proportional hazards model was performed for each baseline factor. Multivariate analysis (stepwise model by backward elimination) was performed with those variables contributing to the univariate model.

\section{RESULTS}

The median follow-up was 32 mo (95\% confidence interval $[\mathrm{CI}], 29-35)$, and the median OS of the entire cohort ( $n=42$; Fig. 1) was 51 mo (95\% CI, 37-65). Twelve patients $(28.6 \%)$ had died by the end of the study. No treatment-related deaths were observed.

The observed treatment response of BMs consisted of $\mathrm{CR}$ in 2 (4.8\%), PR in 14 (33.3\%), MR in 5 (11.9\%), stable disease in $16(38.1 \%)$, and PD in $5(11.9 \%)$ patients. From the 11 patients with bone metastatic pain, $6(55 \%)$ had complete and $5(45 \%)$ partial resolution of symptoms. Median time to progression of BMs was 35 mo $(95 \% \mathrm{CI}$, 26-44) from start of treatment; Figure 1 shows the respective Kaplan-Meier curve of the entire patient cohort.

Looking at the different baseline characteristics with regard to treatment response of BMs (Table 1), none of the examined variables were associated with an increased or decreased rate of regression (CR, PR, or MR). The association with the rate of treatment failure, that is, progression despite treatment, could not be determined because of the small PD group $(n=5)$, but again none of the baseline factors was significant.

PRRT-induced regression of BMs (Fig. 2 illustrates an example case) in turn is associated with a trend toward improved outcome (Fig. 3). The regression group has not yet reached median OS after 53 mo of follow-up (with $71.4 \%$ of patients alive), whereas the median OS of the nonregression group is $39 \mathrm{mo}(95 \% \mathrm{CI}, 33-45 ; P=$ 0.076 , log-rank test).

Risk factors for early progression of BMs after PRRT in the univariate analyses are a $\mathrm{Ki}-67$ index greater than $10 \%$ and a chromogranin A level greater than $600 \mathrm{ng} / \mathrm{mL}$ (Table 2 ). Multivariate analysis leaves none of these 2 variables significant; however, Ki-67 index reaches borderline significance, with a hazard ratio (HR) of 3.4 (95\% CI, 0.9-13.7; $P=0.083)$. The Kaplan-Meier curves, after dichotomizing for chromogranin A (Fig. 4) and Ki-67 index (Fig. 5), illus- 


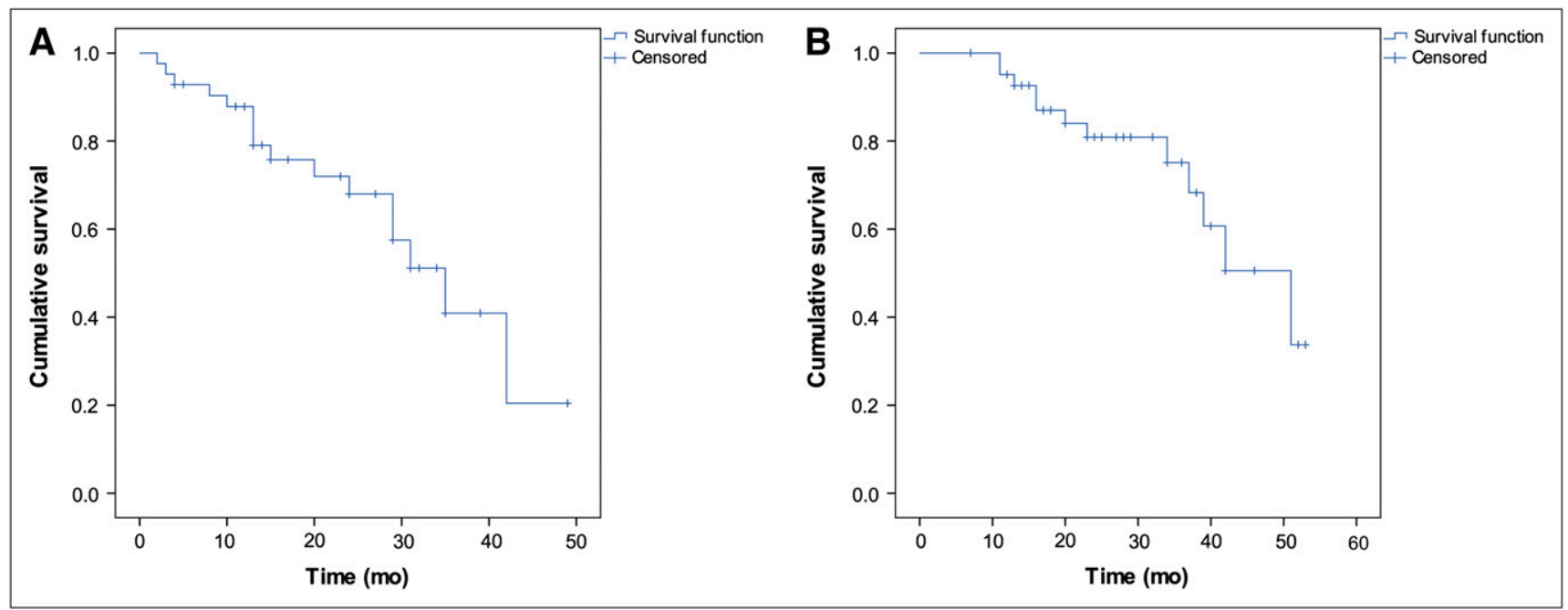

FIGURE 1. Kaplan-Meier curves for osseous progression-free survival (A) and OS (B) of entire study cohort $(n=42)$. Median progressionfree survival was $35 \mathrm{mo}(95 \% \mathrm{Cl}, 26-44)$ and median OS $51 \mathrm{mo}(95 \% \mathrm{Cl}, 37-65)$.

trate the prognostic value of these variables with regard to progression.

\section{DISCUSSION}

This retrospective study shows that BM of well-differentiated GEP NETs treated with PRRT can be controlled effectively over a long period. We found a median time to progression of approximately 3 y ( $35 \mathrm{mo})$, with a median OS in these advanced metastatic patients of $51 \mathrm{mo}$. Our findings support the few retrospective data existing on this GEP NET subgroup: in the largest study on PRRT in GEP NETs (21), Kwekkeboom et al. reported outcome results for 310 patients treated with ${ }^{177} \mathrm{Lu}$-octreotate, of which 68 had BMs. Median OS in this specific subgroup was reported to be 37 mo from start of treatment; further information, such as response or time to progression of BMs, is not available.

In our study, regression of BMs after PRRT as assessed by functional imaging is common (50\% of all cases) and associated with a trend toward improved outcome. Patients in whom osseous tumor regression was achieved did not reach median OS after 53 mo, whereas median OS in the remaining patient group was 39 mo (3-y OS, $89.3 \%$ vs. $63.2 \%$, respectively). The insignificant $P(0.076)$ could be explained by an insufficient follow-up period in this particular setting with few events. However, any observation of improved survival in responding patients has to be viewed with particular caution because of the retrospective nature of the study.

BMs can cause pain, with a significant impact on quality of life. Few data are available for their symptomatic relevance in NET (9); in our cohort, 11 (26\%) patients had metastatic bone pain. In these patients, treatment with PRRT led to complete (55\%) or partial (45\%) resolution of symptoms. The onset of pain alleviation was typically noted within a few weeks after the first treatment cycle, and the duration paralleled time to progression. This palliative potential is notable and relevant in the management of bone metastatic NET.

Analogous to the fact that well-responding GEP NETs exhibit a shorter time to progression $(10,22)$, our retrospective study shows that osseous responders tend to catch up

TABLE 1

Response of BMs to PRRT According to Various Baseline Characteristics

\begin{tabular}{lccc}
\hline Variable & $n$ & Regression & $P$ \\
\hline Total & 42 & $21(50)$ & \\
$\quad$ Tumor type & & & \\
$\quad$ Pancreatic NET & 12 & $7(58.3)$ & 0.734 \\
$\quad$ Other GEP NETs & 30 & $14(46.6)$ & \\
Performance status & & & \\
$\quad$ Karnofsky performance & 12 & $7(58.3)$ & 0.734 \\
$\quad$ score $\leq 70$ & & & \\
$\quad$ Karnofsky performance & 30 & $14(46.6)$ & \\
$\quad$ score $>70$ & & & \\
$\quad$ Chromogranin A & & & \\
$\quad \leq 600$ ng/mL & 26 & $16(61.5)$ & 0.111 \\
$\quad>600$ ng/mL & 16 & $5(31.2)$ & \\
Neuron-specific enolase & & & \\
$\quad \leq 15$ ng/mL & 17 & $9(52.9)$ & 1 \\
$\quad>15$ ng/mL & 25 & $12(48.0)$ & \\
Ki-67 index & & & \\
$\quad \leq 10 \%$ & 33 & $17(51.6)$ & 1 \\
$\quad>10 \%$ & 9 & $4(44.4)$ & \\
No. of lesions & & & \\
$\quad$ Few $(\leq 10)$ & 26 & $13(50)$ & 1 \\
$\quad$ Multiple & 16 & $8(50)$ & \\
Tracer uptake & & & \\
$\quad \leq$ Grade 2 & 20 & $7(35)$ & 0.121 \\
$\quad>$ Grade 2 & 22 & $14(63.6)$ & \\
$\quad$ & &
\end{tabular}

Regression is CR, PR, or MR.

Data in parentheses are percentages. 


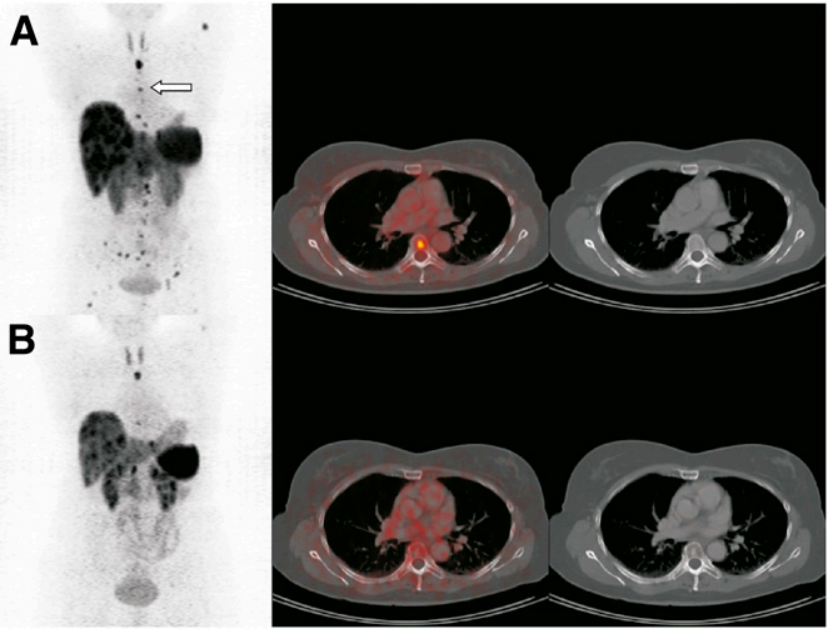

FIGURE 2. Regression of BMs illustrated by ${ }^{68} \mathrm{Ga}-\mathrm{DOTATOC}$ PET/ CT before (A) and 3 mo after (B) PRRT in a patient with metastatic midgut carcinoid. Maximum-intensity-projection PET images (coronal views) are shown on left, fused and unfused CT images (selected lesion indicated by arrow) on right. This patient had functional remission of BMs accompanied by osteosclerotic changes, classified as PR. Patient remained in remission for 34 mo by end of study.

with nonresponders regarding progression-free survival after a certain interval (Fig. 3A). Although pancreatic NET are known to respond better to PRRT than GEP NETs of other origin (carcinoid tumors) according to morphologic (extraosseous) response criteria such as those of the World Health Organization, RECIST, or SWOG $(10,22)$, we could not find a corresponding difference in response of BM. The regression rate was similar in pancreatic $(58.3 \%)$ and nonpancreatic $(46.6 \%)$ GEP NETs $(P=0.734)$. Also, progression-free survival of pancreatic NET was not significantly different from that of nonpancreatic NET, in our bone metastatic study cohort (29 vs. 35 mo, respectively). Although caution is required when interpreting retrospective results of a limited cohort $(n=42)$, this comparable outcome of
BMs in pancreatic and nonpancreatic GEP tumors is noteworthy and may help to optimize patient management and scheduling of follow-up.

The only baseline variables contributing to progressionfree survival in the univariate Cox proportional hazards model were the tumor marker level (chromogranin A $>600$ ng/mL; HR, 3.2 [95\% CI, 1.1-9.5]; $P=0.039$ ) and the proliferation rate (Ki-67 index $>10 \%$; HR, $5.2[95 \% \mathrm{CI}$, $1.5-18.0] ; P=0.01)$. However, none of these proved significant on multivariate analysis, probably because of the small sample size. The chromogranin A level, which to some extent reflects overall tumor burden, has been found in some studies to be a negative predictor of outcome $(4,23)$. Our finding confirms that a high chromogranin A level is a negative predictor for the particular setting of PRRT in the presence of BMs. However, multifocality of BMs, more specifically reflecting osseous tumor load than chromogranin A levels, was not a negative predictive factor of outcome after PRRT. This retrospective factor analysis should be viewed with caution in light of the relatively small number of the study population. This is also illustrated by the fact that the known predictive factor of tumor uptake $(11,21)$, which can be seen as a surrogate for tumor absorbed dose, failed to show a significant impact on tumor regression in our analysis $(P=0.12)$.

The proliferation index has also been shown to affect outcome in the literature $(6,24)$, which is not unexpected considering its biologic meaning. Our data confirm the propensity toward earlier progression with increased Ki-67 indices in our cohort of radiopeptide-treated BMs, irrespective of the initial treatment response recorded. To the best of our knowledge, there are no data available for comparison because no studies have evaluated the response and progression of BM of NET in the setting of any treatment modality. The parameter Ki-67 index is limited; it is rarely determined on BMs and may yield different results depending on the site and time point at which it is assessed.
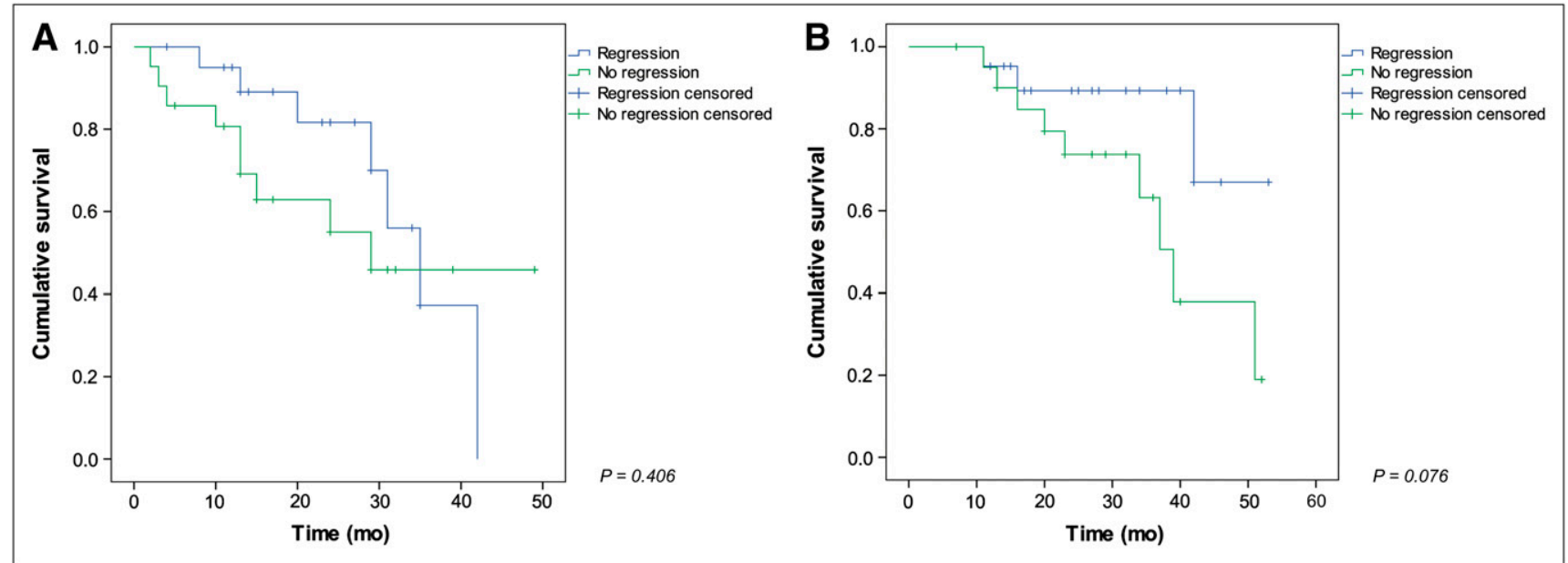

FIGURE 3. Patients showing regression of BMs after PRRT had similar progression-free survival (A) but trend toward prolonged OS (B) when compared with nonresponders (log-rank test, $P=0.076$ ). 
TABLE 2

Uni- and Multivariate Analyses for Potential Factors Associated with Time to Progression of BMs

\begin{tabular}{|c|c|c|c|c|c|c|c|c|}
\hline \multirow[b]{2}{*}{ Variable } & \multicolumn{2}{|c|}{ Progression-free survival } & \multicolumn{3}{|c|}{ Univariate analysis } & \multicolumn{3}{|c|}{ Multivariate analysis } \\
\hline & Median (mo) & $95 \% \mathrm{Cl}$ & $\mathrm{HR}$ & $95 \% \mathrm{Cl}$ & $P$ & $\mathrm{HR}$ & $95 \% \mathrm{Cl}$ & $P$ \\
\hline Total & 35 & $26-44$ & & & & & & \\
\hline \multicolumn{9}{|l|}{ Age } \\
\hline$\leq 65$ y & 35 & $16-54$ & & & & & & \\
\hline$>65$ y & 29 & 19-39 & - & & 0.823 & & & \\
\hline \multicolumn{9}{|l|}{ Tumor type } \\
\hline Pancreatic NET & 29 & $18-40$ & - & & 0.497 & & & \\
\hline Other GEP NETs & 35 & $16-54$ & & & & & & \\
\hline \multicolumn{9}{|l|}{ Performance status } \\
\hline Karnofsky performance score $\leq 70$ & 29 & $18-40$ & - & & 0.629 & & & \\
\hline Karnofsky performance score $>70$ & 35 & $26-44$ & & & & & & \\
\hline \multicolumn{9}{|l|}{ Chromogranin A } \\
\hline$\leq 600 \mathrm{ng} / \mathrm{mL}$ & 42 & $31-53$ & & & & & & \\
\hline$>600 \mathrm{ng} / \mathrm{mL}$ & 24 & $12-36$ & 3.2 & $1.1-9.5$ & 0.039 & 2.1 & $0.6-7.6$ & 0.245 \\
\hline \multicolumn{9}{|l|}{ Neuron-specific enolase } \\
\hline$\leq 15 \mathrm{ng} / \mathrm{mL}$ & 35 & $8-62$ & & & & & & \\
\hline$>15 \mathrm{ng} / \mathrm{mL}$ & 31 & $25-37$ & - & & 0.708 & & & \\
\hline \multicolumn{9}{|l|}{ Ki-67 index } \\
\hline$\leq 10 \%$ & 35 & $26-44$ & & & & & & \\
\hline$>10 \%$ & 15 & $6-24$ & 5.2 & $1.5-18.0$ & 0.01 & 3.4 & $0.9-13.7$ & 0.083 \\
\hline \multicolumn{9}{|l|}{ No. of lesions } \\
\hline Few $(\leq 10)$ & 31 & $19-43$ & & & & & & \\
\hline Multiple & 35 & $19-51$ & - & & 0.773 & & & \\
\hline \multicolumn{9}{|l|}{ Tracer uptake } \\
\hline$\leq$ Grade 2 & 42 & $23-61$ & & & & & & \\
\hline$>$ Grade 2 & 29 & 22-36 & - & & 0.314 & & & \\
\hline
\end{tabular}

Interestingly, the number of metastatic bone lesions that reflect osseous tumor burden as well as the Karnofsky performance score did not affect osseous tumor regression induced by PRRT (Table 1). Also, these 2 factors did not affect progression-free survival (Table 2). This finding is encouraging, because it might indicate that patients with advanced BMs and a reduced performance score may respond and benefit from PRRT in a way similar to patients with only a few $(<10)$ bone lesions. Although comparable investigations on BM in the literature are lacking, analogous analyses for liver metastases have linked high tumor load and low performance status to poor outcome $(21,25,26)$.

The main limitation of the study is the population size regarding statistical analyses of smaller subgroups. Particularly, the group of patients progressing despite PRRT (PD group; $n=5$ ) was too small to permit valid analyses of this factor. Also, multivariate analysis should be viewed with
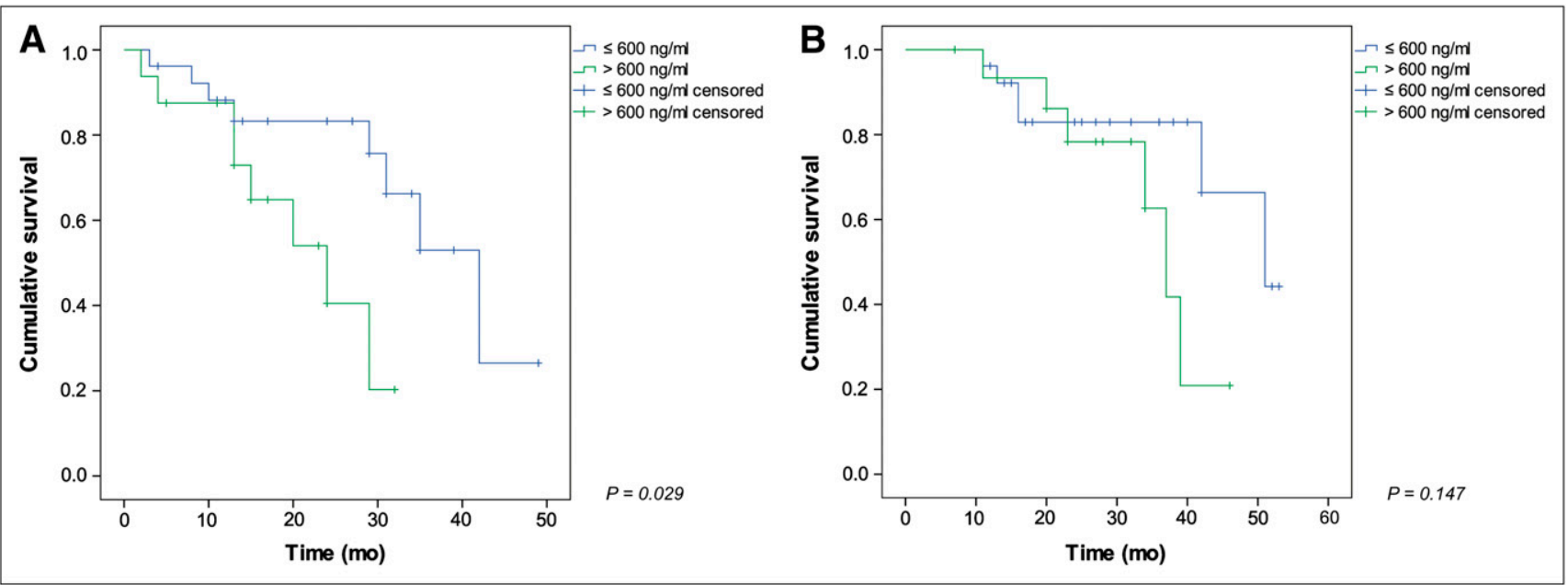

FIGURE 4. Progression-free survival (A) and OS (B) stratified by pretreatment chromogranin A plasma level (cutoff, $600 \mathrm{ng} / \mathrm{mL}$ ). 


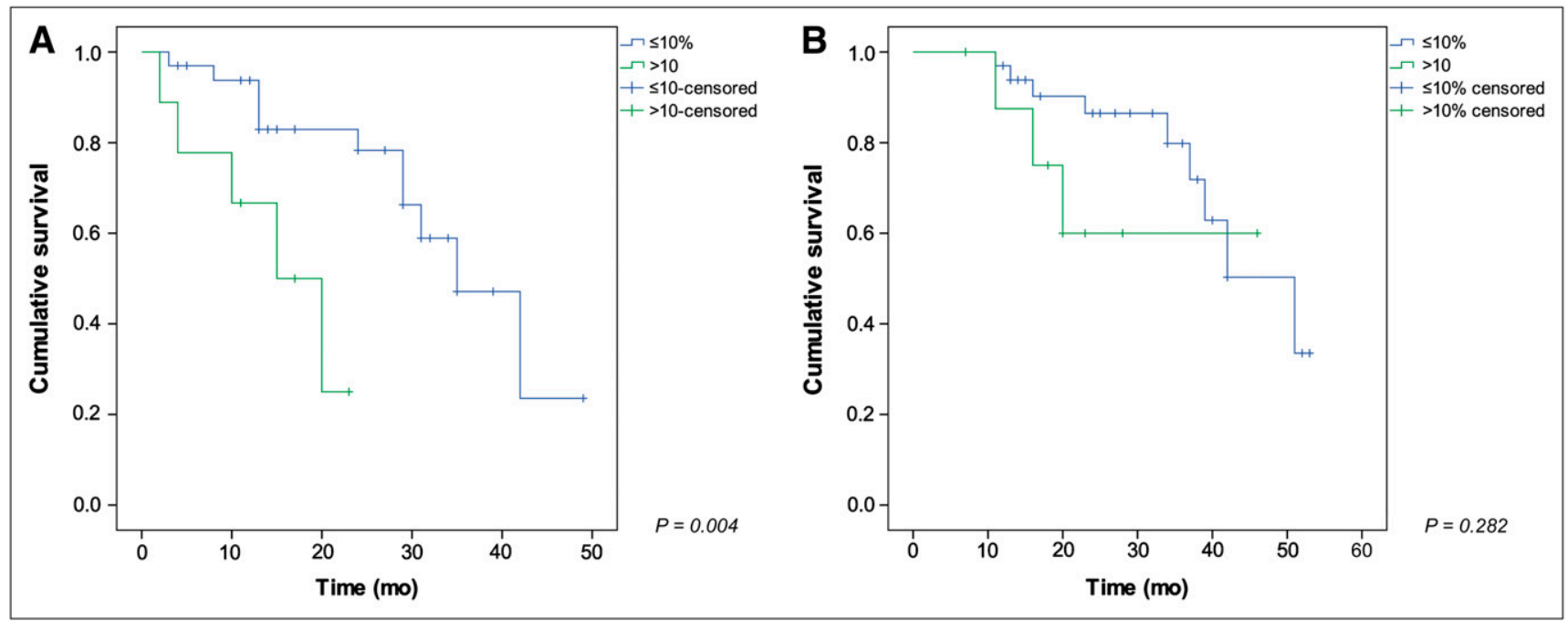

FIGURE 5. Progression-free survival (A) and OS (B) stratified by tumor proliferation index (cutoff, 10\%).

caution in this respect. Strong predictors for negative treatment outcome after PRRT would be expected to become clear despite these caveats, even in this limited study cohort. However, as mentioned earlier in the discussion, one important predictive factor of treatment response, the grade of tumor uptake, failed to show significance for response in this analysis. The presented population, a group of 42 patients with BMs undergoing identical treatment, is an important and valuable study cohort for analyses in this rare entity. We recognize the additional limitation of the retrospective study design and its negative impact on the strength of our conclusions.

\section{CONCLUSION}

Our study shows that BM of well-differentiated gastroenteropancreatic NET are effectively controlled by PRRT, leading to long progression-free and overall survival as well as alleviation of pain if present. Treatment efficacy - that is, response and long-term inhibition of progression-is not clearly affected by multifocality of bone lesions or reduced patient condition. This may encourage the use of PRRT in advanced bone metastatic disease; however, larger studies are needed to confirm and expand these initial findings.

\section{DISCLOSURE STATEMENT}

The costs of publication of this article were defrayed in part by the payment of page charges. Therefore, and solely to indicate this fact, this article is hereby marked "advertisement" in accordance with 18 USC section 1734.

\section{ACKNOWLEDGMENTS}

We thank Professors Eric Krenning and Dik Kwekkeboom (Erasmus Medical Center, Rotterdam, Netherlands) for sharing their invaluable experience in the receptor-targeting field and making somatostatin receptor-mediated treatment at all possible in Bonn. Also, we thank the personnel of the Nuclear Medicine Department and especially the nursing staff of the therapy ward and Ulrike Kuhn-Seifer. No potential conflict of interest relevant to this article was reported.

\section{REFERENCES}

1. Lebtahi R, Cadiot G, Delahaye N, et al. Detection of bone metastases in patients with endocrine gastroenteropancreatic tumors: bone scintigraphy compared with somatostatin receptor scintigraphy. J Nucl Med. 1999;40:1602-1608.

2. Panzuto F, Nasoni S, Falconi M, et al. Prognostic factors and survival in endocrine tumor patients: comparison between gastrointestinal and pancreatic localization. Endocr Relat Cancer. 2005;12:1083-1092.

3. Ezziddin S, Logvinski T, Yong-Hing $\mathrm{C}$, et al. Factors predicting tracer uptake in somatostatin receptor and MIBG scintigraphy of metastatic gastroenteropancreatic neuroendocrine tumors. $J$ Nucl Med. 2006;47:223-233.

4. Durante C, Boukheris H, Dromain C, et al. Prognostic factors influencing survival from metastatic (stage IV) gastroenteropancreatic well-differentiated endocrine carcinoma. Endocr Relat Cancer. 2009;16:585-597.

5. Meijer WG, van der Veer E, Jager PL, et al. Bone metastases in carcinoid tumors: clinical features, imaging characteristics, and markers of bone metabolism. $J$ Nucl Med. 2003;44:184-191.

6. Pape UF, Berndt U, Muller-Nordhorn J, et al. Prognostic factors of long-term outcome in gastroenteropancreatic neuroendocrine tumours. Endocr Relat Cancer. 2008;15:1083-1097.

7. Gibril F, Doppman JL, Reynolds JC, et al. Bone metastases in patients with gastrinomas: a prospective study of bone scanning, somatostatin receptor scanning, and magnetic resonance image in their detection, frequency, location, and effect of their detection on management. J Clin Oncol. 1998;16:1040-1053.

8. Putzer D, Gabriel M, Henninger B, et al. Bone metastases in patients with neuroendocrine tumor: ${ }^{68} \mathrm{Ga}$-DOTA-Tyr3-octreotide PET in comparison to CT and bone scintigraphy. J Nucl Med. 2009;50:1214-1221.

9. Kos-Kudla B, O'Toole D, Falconi M, et al. ENETS consensus guidelines for the management of bone and lung metastases from neuroendocrine tumors. Neuroendocrinology. 2010;91:341-350.

10. Kwekkeboom DJ, Mueller-Brand J, Paganelli G, et al. Overview of results of peptide receptor radionuclide therapy with 3 radiolabeled somatostatin analogs. J Nucl Med. 2005;46(suppl 1):62S-66S.

11. Kwekkeboom DJ, de Herder WW, van Eijck CH, et al. Peptide receptor radionuclide therapy in patients with gastroenteropancreatic neuroendocrine tumors. Semin Nucl Med. 2010;40:78-88.

12. van Essen M, Krenning EP, Kam BL, de Herder WW, Feelders RA, Kwekkeboom DJ. Salvage therapy with ${ }^{177} \mathrm{Lu}$-octreotate in patients with bronchial and gastroenteropancreatic neuroendocrine tumors. J Nucl Med. 2010;51:383-390. 
13. Forrer F, Uusijarvi H, Storch D, Maecke HR, Mueller-Brand J. Treatment with ${ }^{177} \mathrm{Lu}$-DOTATOC of patients with relapse of neuroendocrine tumors after treatment with ${ }^{90}$ Y-DOTATOC. J Nucl Med. 2005;46:1310-1316.

14. Rindi G, Kloppel G, Alhman H, et al. TNM staging of foregut (neuro)endocrine tumors: a consensus proposal including a grading system. Virchows Arch. 2006;449:395-401.

15. Rindi G, Kloppel G, Couvelard A, et al. TNM staging of midgut and hindgut (neuro) endocrine tumors: a consensus proposal including a grading system. Virchows Arch. 2007;451:757-762.

16. Breeman WA, van der Wansem K, Bernard BF, et al. The addition of DTPA to $\left[{ }^{177} \mathrm{Lu}\right.$-DOTA0,Tyr3]octreotate prior to administration reduces rat skeleton uptake of radioactivity. Eur J Nucl Med Mol Imaging. 2003;30:312-315.

17. Breeman WA, De Jong M, Visser TJ, Erion JL, Krenning EP. Optimising conditions for radiolabelling of DOTA-peptides with ${ }^{90} \mathrm{Y},{ }^{111} \mathrm{In}$ and ${ }^{177} \mathrm{Lu}$ at high specific activities. Eur J Nucl Med Mol Imaging. 2003;30:917-920.

18. Green S, Weiss GR. Southwest Oncology Group standard response criteria, endpoint definitions and toxicity criteria. Invest New Drugs. 1992;10:239-253.

19. Ezziddin S, Opitz M, Attassi M, et al. Impact of the Ki-67 proliferation index on response to peptide receptor radionuclide therapy. Eur J Nucl Med Mol Imaging. 2011;38:459-466.

20. Costelloe CM, Chuang HH, Madewell JE, Ueno NT. Cancer response criteria and bone metastases: RECIST 1.1, MDA and PERCIST. J Cancer. 2010;1:80-92.
21. Kwekkeboom DJ, de Herder WW, Kam BL, et al. Treatment with the radiolabeled somatostatin analog [177 Lu-DOTA 0,Tyr3]octreotate: toxicity, efficacy, and survival. J Clin Oncol. 2008;26:2124-2130.

22. Kwekkeboom DJ, Bakker WH, Kam BL, et al. Treatment of patients with gastroentero-pancreatic (GEP) tumours with the novel radiolabelled somatostatin analogue [ ${ }^{177}$ Lu-DOTA(0),Tyr3]octreotate. Eur J Nucl Med Mol Imaging. 2003; 30:417-422.

23. Clancy TE, Sengupta TP, Paulus J, Ahmed F, Duh MS, Kulke MH. Alkaline phosphatase predicts survival in patients with metastatic neuroendocrine tumors. Dig Dis Sci. 2006;51:877-884.

24. Strosberg J, Nasir A, Coppola D, Wick M, Kvols L. Correlation between grade and prognosis in metastatic gastroenteropancreatic neuroendocrine tumors. Hum Pathol. 2009;40:1262-1268.

25. Valkema R, Pauwels S, Kvols LK, et al. Survival and response after peptide receptor radionuclide therapy with $\left[{ }^{90}\right.$ Y-DOTA0,Tyr3]octreotide in patients with advanced gastroenteropancreatic neuroendocrine tumors. Semin Nucl Med. 2006;36:147-156.

26. Kwekkeboom DJ, Kam BL, van Essen M, et al. Somatostatin-receptor-based imaging and therapy of gastroenteropancreatic neuroendocrine tumors. Endocr Relat Cancer. 2010;17:R53-R73. 